\title{
Effects of Flow Parameters on the Stability of Conical Liquid Sheets FU Qingfei ${ }^{1, a}$, DU Minglong ${ }^{1, b}$
}

${ }^{1}$ School of Astronautics, Beijing University of Aeronautics and Astronautics, Beijing, China.100191 afuqingfei@buaa.edu.cn, ${ }^{\mathrm{b}} \mathrm{dml} 1314521 @ 126 . c 0 m$

Keywords: Atomization; Linear stability; Conical liquid sheet; Flow parameter

\begin{abstract}
Linear instability theory can evaluate the beginnings of instability in a liquid sheet. It provides a good starting point for predicting unstable, wave-type behavior in a liquid sheet with disturbances. An existing linear instability analysis method was used to investigate the breakup of a conical sheet formed by a pressure swirl injector. The dispersion relation for the conical sheet was solved numerically. The effect of flow parameters and spray condition on the linear stability of conical sheet is mainly discussed.
\end{abstract}

\section{Introduction}

Pressure swirl injectors are widely used in the preparation for the combustion mixtures of high energy release devices, such as rocket and jet engines. In a swirl injector, angular momentum is imposed on the liquid to form a swirling motion using tangential inlets or cylindrical swirl insert. Under the action of centrifugal force, the liquid spreads out in the form of a conical sheet as soon as it leaves the nozzle. Understanding the factors that influence the disintegration process of liquid sheet will benefit not only atomizer design and improvement but also numerical simulation of spray combustion. The breakup and atomization mechanism of the spray film from an injector has always been a fundamental study task. Although the breakup mechanism for a flat liquid sheet has been understood for some time, a theory for the breakup mechanism of conical sheets has yet to be developed.

It is well known that the disintegration of the liquid sheet is due to the growth of unstable waves at the interface between the gas and the liquid sheet. Various forces, such as aerodynamic force, inertial force, surface tension, shear force and centrifugal force, involved in the disintegration process, compete to dominate the instability of the liquid sheet. Extensive theoretical and experimental studies have been conducted on the instability of planar liquid sheets and annular sheets. In the 1970s, Lin et al. [1] studied the atomization mechanism of a columnar jet in both a temporal model and a convective model. Crapper, Dombroski and Pyott [2] studied the linear stability of an annular liquid sheet in 1975 but they did not research the liquid sheet cone formed by swirl injector. Fraser et al. [3] investigated the breakup process of a conical liquid sheet. Yue and Yang [4] made an investigation on the spatial instability of a conical sheet, whose method is similar to Crapper's. Briffa and Dombrowski [5] and Dombrowski and Hooper [6] correlated the breakup length of conical liquid sheets on the basis of theoretical relation developed from the first order theory of Squire [7].

In this paper, a linear stability model is applied to investigate the stability of the conical liquid sheet. The effects of several flow parameters on the stability of conical liquid sheets are discussed.

\section{Linear Stability Analysis for Conical Liquid Sheet}

Linear sheet instability analyses have been performed by many researchers in the past several decades. However, most of these studies concerned a planar sheet or an annular sheet; the conical liquid sheet has not been studied adequately in previous work. For this reason, results for this case are presented herein.

For a model of a conical liquid sheet, and the derivation of dispersion relationship refer to reference [2]. We consider a conical sheet with internal radius a and thickness h, as shown in Fig. 1. $\mathrm{a} 0$ and h0 denote the initial sheet thickness and the radius of injector spout respectively. $\beta$ denotes the 
half of the spray cone angle. In the undisturbed state, the liquid in the sheet, of density $\rho_{l}$, has velocity $V$, and the surrounding gas, of density $\rho_{g}$, is at rest. The surface tension of liquid is $\sigma$.

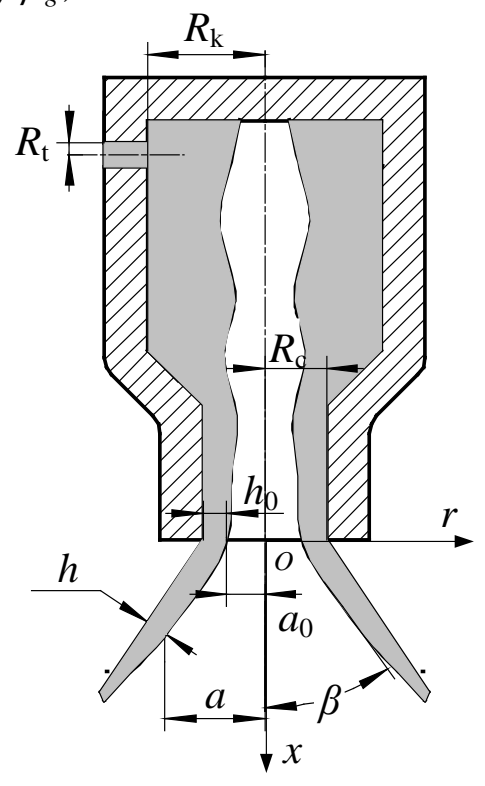

Fig. 1 Schematic of a conical liquid sheet

Following the usual procedure, the displacement of the inner and outer surface of liquid sheet may be taken respectively to be

$$
\left.\begin{array}{l}
\eta_{1}=R \eta_{1,0} \exp [i(k x-\bar{\omega} t)] \\
\eta_{2}=R \eta_{2,0} \exp [i(k x-\bar{\omega} t)]
\end{array}\right\}
$$

where $\eta$ denotes the fluctuation amplitude, subscript 1 , 2 represent the inner and outer surface of the liquid sheet, subscript 0 means the initial state. Wave number $k=2 \pi / \lambda$, where $\lambda$ is wavelength of disturbance wave. $\bar{\omega}=\bar{\omega}_{r}+i \bar{\omega}_{i}$, where real part $\bar{\omega}_{r}$ denotes disturbance frequency, imaginary part $\bar{\omega}_{i}$ denotes the growth rate of disturbance wave.

The detailed dispersion relation for a conical liquid sheet refers to Yue and Yang [4].

\section{Results and Discussion}

Influence of cone angle. Fig. 2 shows the influence of the half spray cone angle $\beta$ on the linear stability of the conical sheets. It is shown that the maximum wave growth rate and optimum wave number decrease in both the varicose model and sinuous model with increasing cone angle. Figure 2 also shows that with increasing cone angle, the sinuous disturbance wave growth rate decreases more slowly than that of the varicose disturbance wave; and there are two peaks in the varicose model. These two wave numbers correspond to two wavelengths, and the growth rate of the short wave is much less than that of the long wave.

Influence of liquid sheet thickness. Fig. 3 illustrates the influence of liquid sheet thickness on the stability of a conical sheet. For the varicose disturbance model, the results show that liquid thickness has no effect on the optimum wave number. But the disturbance wave growth rate increases with the increase of liquid sheet thickness, i.e., liquid sheet disintegrates more quickly because increased liquid sheet thickness means decreasing the surface tension of the liquid sheet, which restrains the liquid sheet from breaking up.

For a sinuous disturbance wave, the maximum wave growth rate decreases with increased liquid sheet thickness. But the optimum wave number decreases slightly, which means the disturbance wave length increases. 

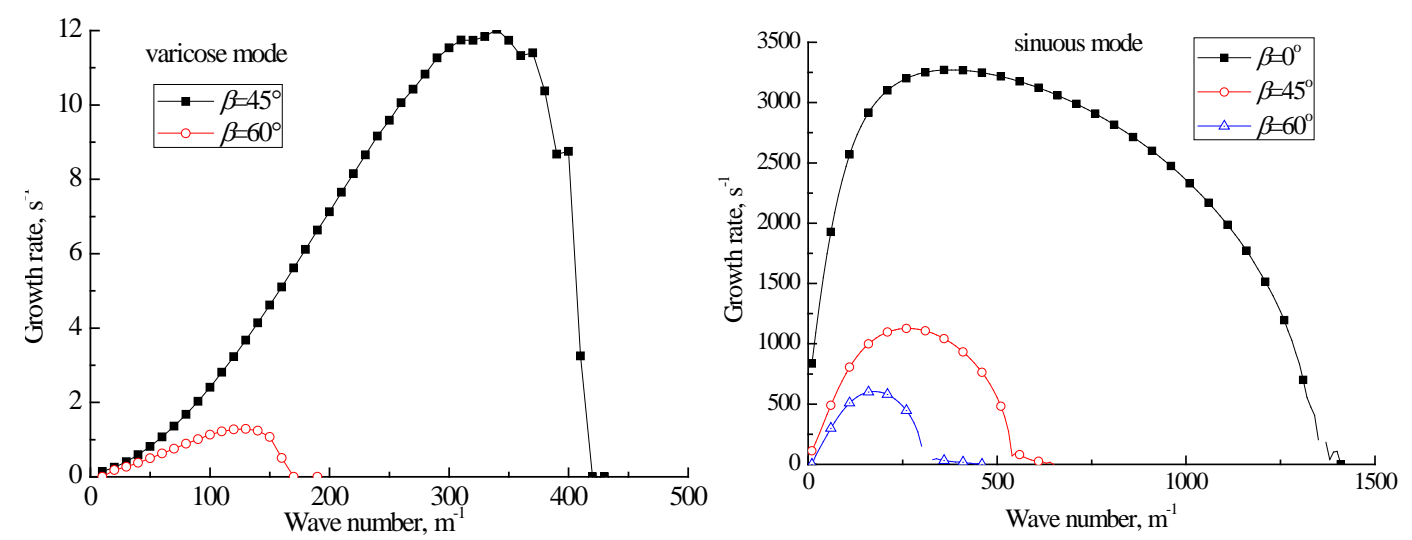

Fig. 2 Effects of $\beta$ on the stability of conical sheet $\left(h_{0}=0.1 \mathrm{~mm}, r_{0}=0.8 \mathrm{~mm}, V=10 \mathrm{~m} / \mathrm{s}\right)$
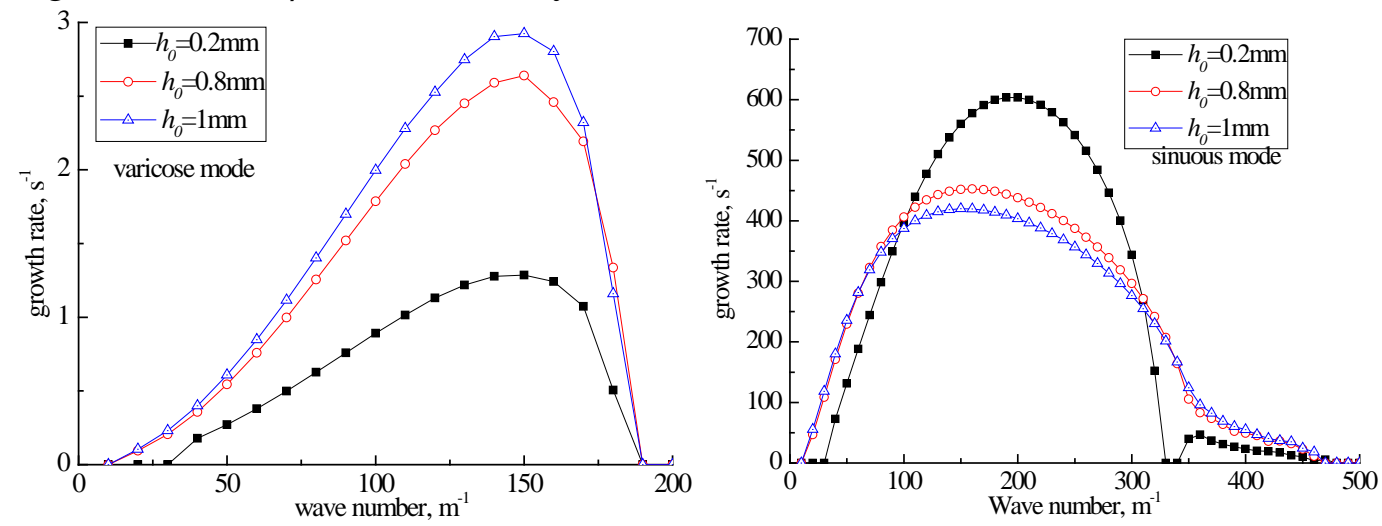

Fig.3 Effects of liquid sheet thickness on the stability of conical sheet $\left(r_{0}=0.8 \mathrm{~mm}, \beta=60^{\circ}, V=10 \mathrm{~m} / \mathrm{s}\right)$

Influence of the radius of the injector spout. The curves in Fig.4 demonstrate the effect of injector spout radius variation on the breakup of a conical sheet. Whether in varicose disturbance model or sinuous model, the maximum growth rate and optimum wave number decrease with increasing $a_{0}$, which indicates that a smaller injector spout is advantageous for breakup of liquid sheet. This agrees with the empirical method.
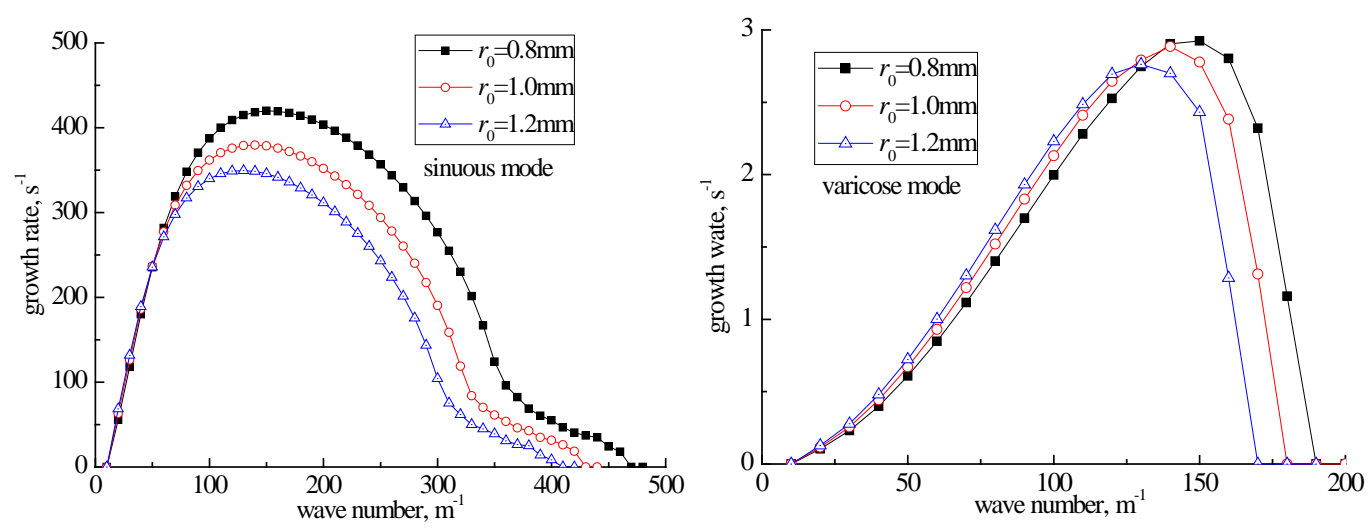

Fig. 4 Effects of spout radius on the stability of conical sheet $\left(h_{0}=0.1 \mathrm{~mm}, \beta=60^{\circ}, V=10 \mathrm{~m} / \mathrm{s}\right.$ )

Influence of spray velocity. Fig.5 illustrates the influence of liquid sheet spray velocity on the linear stability of a conical sheet. For both varicose and sinuous disturbance models, maximum growth rate and optimum wave number increase greatly with increasing sheet spray velocity. This indicates that liquid sheet spray velocity promotes breakup of a conical liquid sheet; increasing the spray velocity depresses the effect of liquid surface tension, and the break up effect of aerodynamic force intensifies. 

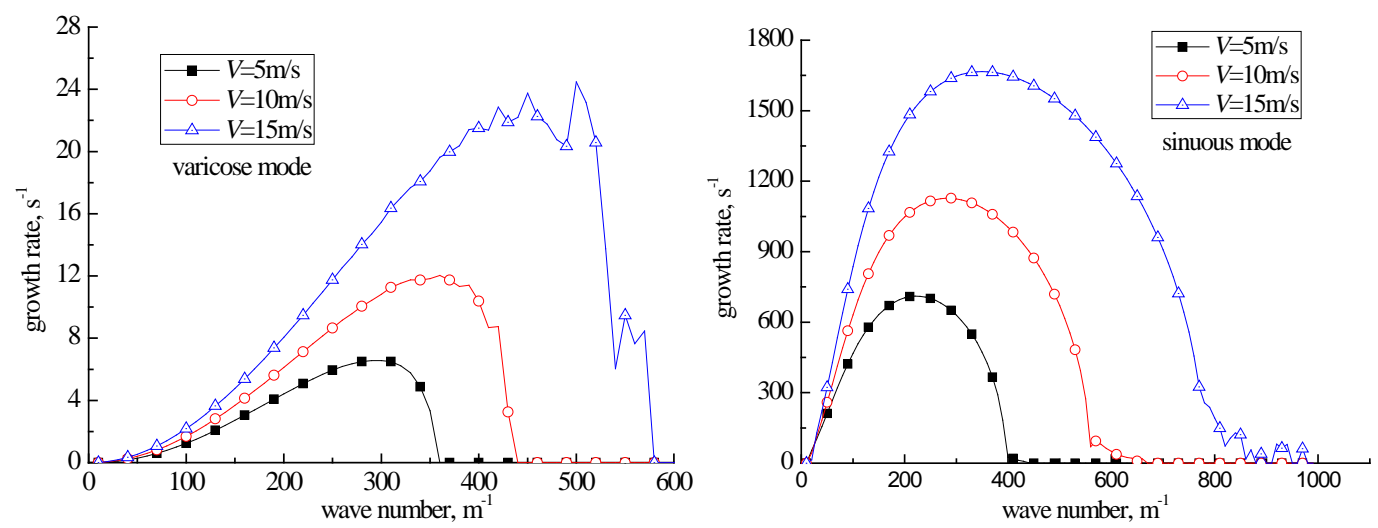

Fig.5 Effects of spray velocity on stability of conical sheet $\left(h_{0}=0.2 \mathrm{~mm}, \beta=45^{\circ}, r_{0}=0.8 \mathrm{~mm}\right)$

\section{Summary}

A linear instability theoretical model is used to predict the instability of a conical liquid sheet. The model incorporates essential features of the liquid sheet downstream of a swirl injector. As the swirl sheet sprays out of the injector, the liquid sheet breaks away from the confines of the injector inner wall and disintegrates to liquid ligaments. A linear instability analysis method was applied to study the breakup of the conical sheet. The calculation results from solving the dispersion relation of the conical sheet are as follows:

(1) Increasing sheet cone angle causes the maximum disturbance wave growth rate and optimum wave number to decrease. The disturbance wave tends to change into a long wave, i.e. increasing spray cone angle is unfavorable for atomization.

(2) For the varicose disturbance model, increasing liquid sheet thickness accelerated the atomization of the conical sheet. For the sinuous disturbance model, the sheet thickness influence rule was reversed.

(3) A thinner spout is better for atomization.

\section{Acknowledgements}

The financial support of National Natural Science Foundation of China (Grant No. 11302013) is gratefully acknowledged.

\section{References}

[1] S.P. Lin, D.J. Kang: Phys. Fluids Vol. 30 (1987), p.2000.

[2] G. D. Crapper, N. Dombrowski, G.A.D. Pyott: J. Fluid Mech. Vol. 68 (1975), p.497.

[3] R.P. Fraser, P. Eisenklam, N. Dombrowski, D. Hasson: A. I. Che. J. Vol. 8 (1962), p. 672.

[4] M. Yue, M. L. Yang: Journal of Aerospace Power Vol. 18 (2003), p. 794.

[5] F. E. J. Briffa, N. Dombrowski: AIChE Journal Vol. 12(1966), p. 708.

[6] N. Dombrowski, P. C. Hooper: Chemical Engineering Science Vol. 17 (1962), p. 291.

[7] H. B. Squire: British Journal of Applied Physics Vol. 4 (1953), p. 167. 